\title{
Characterization of revenue equivalence
}

Citation for published version (APA):

Heydenreich, B., Müller, R. J., Uetz, M. J., \& Vohra, R. (2008). Characterization of revenue equivalence. METEOR, Maastricht University School of Business and Economics. METEOR Research Memorandum No. 001 https://doi.org/10.26481/umamet.2008001

Document status and date:

Published: 01/01/2008

DOI:

10.26481/umamet.2008001

Document Version:

Publisher's PDF, also known as Version of record

\section{Please check the document version of this publication:}

- A submitted manuscript is the version of the article upon submission and before peer-review. There can be important differences between the submitted version and the official published version of record.

People interested in the research are advised to contact the author for the final version of the publication, or visit the DOI to the publisher's website.

- The final author version and the galley proof are versions of the publication after peer review.

- The final published version features the final layout of the paper including the volume, issue and page numbers.

Link to publication

\footnotetext{
General rights rights.

- You may freely distribute the URL identifying the publication in the public portal. please follow below link for the End User Agreement:

www.umlib.nl/taverne-license

Take down policy

If you believe that this document breaches copyright please contact us at:

repository@maastrichtuniversity.nl

providing details and we will investigate your claim.
}

Copyright and moral rights for the publications made accessible in the public portal are retained by the authors and/or other copyright owners and it is a condition of accessing publications that users recognise and abide by the legal requirements associated with these

- Users may download and print one copy of any publication from the public portal for the purpose of private study or research.

- You may not further distribute the material or use it for any profit-making activity or commercial gain

If the publication is distributed under the terms of Article $25 \mathrm{fa}$ of the Dutch Copyright Act, indicated by the "Taverne" license above, 
Birgit Heydenreich, Rudolf Müller, Marc Uetz, Rakesh Vohra

Characterization of Revenue Equivalence

$\mathrm{RM} / 08 / 001$

(RM/07/017 -revised-)

JEL code: D71, C72, C69

\section{METEबR

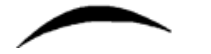

Maastricht research school of Economics

of TEchnology and ORganizations

Universiteit Maastricht

Faculty of Economics and Business Administration P.O. Box 616

NL - 6200 MD Maastricht

phone : ++31433883830

fax $\quad$ : ++31433884873 


\title{
Characterization of Revenue Equivalence
}

\author{
Birgit Heydenreich ${ }^{1} \quad$ Rudolf Müller ${ }^{1} \quad$ Marc Uetz $^{2} \quad$ Rakesh Vohra $^{3}$
}

January 10, 2008

\begin{abstract}
The property of an allocation rule to be implementable in dominant strategies by a unique payment scheme is called revenue equivalence. In this paper we give a characterization of revenue equivalence based on a graph theoretic interpretation of the incentive compatibility constraints. The characterization holds for any (possibly infinite) outcome space and many of the known results are immediate consequences. Moreover, revenue equivalence can be identified in cases where existing theorems are silent.
\end{abstract}

\section{Introduction}

One of the most important results of auction theory is the Revenue Equivalence Theorem. Subject to certain reasonable assumptions, it concludes that a variety of different auctions generate the same expected revenue for the seller. Klemperer (1999) writes that "much of auction theory can be understood in terms of this theorem.....". Hence the long line of papers that have attempted to relax the sufficient conditions under which revenue equivalence holds. The present paper provides necessary and sufficient conditions on the underlying primitives for revenue equivalence to hold.

\footnotetext{
${ }^{1}$ Maastricht University, Quantitative Economics, P.O.Box 616, 6200 MD Maastricht, The Netherlands. Email:\{b.heydenreich,r.muller\}@ke.unimaas.nl

The first author is supported by NWO grant 2004/03545/MaGW 'Local Decisions in Decentralised Planning Environments'.

${ }^{2}$ University of Twente, Applied Mathematics, P.O. Box 217, 7500 AE Enschede, The Netherlands. Email: m.uetz@utwente.nl

${ }^{3}$ Northwestern University, Department of Managerial Economics and Decision Sciences, Kellogg Graduate School of Management, Evanston IL 60208. Email: r-vohra@kellogg.northwestern.edu
} 
We consider direct revelation mechanisms for agents with multidimensional types. Such mechanisms consist of an allocation rule and a payment scheme. The allocation rule selects an outcome depending on the agents' reported types, whereas the payment scheme assigns a payment to every agent. We focus attention on allocation rules that are implementable in dominant strategies. Hereafter we refer to such rules as implementable. In this environment we characterize the uniqueness of the relevant payment scheme in terms of conditions that are easily verified in potential applications. The property of an allocation rule to be implementable in dominant strategies by a unique payment scheme is called revenue equivalence. Our characterization of revenue equivalence is based on a graph theoretic interpretation of the incentive compatibility constraints. This interpretation has been used before by Rochet (1987), Gui, Müller, and Vohra (2004), as well as Saks and Yu (2005) to identify allocation rules that are implementable in dominant strategies. Müller, Perea, and Wolf (2007) used it to identify Bayes-Nash implementable allocation rules. With this graph theoretic interpretation, our characterizing condition for revenue equivalence is almost self-evident and the proof writes itself. The characterization holds for any (possibly infinite) outcome space. Many of the known results about revenue equivalence are immediate consequences of our characterization. More importantly, with this characterization revenue equivalence can be identified in cases where existing theorems are silent.

Related Work. The bulk of prior work on revenue equivalence has been devoted to identifying sufficient conditions on the type space that ensure all allocation rules from a given class satisfy revenue equivalence. The papers by Green and Laffont (1977) and Holmström (1979) restrict attention to allocation rules called 'utilitarian maximizers', that is, allocation rules that maximize the sum of the valuations of all agents. Holmström (1979), generalizing the paper by Green and Laffont (1977), shows that when the type space is smoothly path connected then utilitarian maximizers satisfy revenue equivalence.

Myerson (1981) shows that revenue equivalence holds for every implementable rule in a setting where the type space is an interval of the real line, the outcome space is a lattice and an agents valuation for an outcome is continuous and supermodular in his type.

Krishna and Maenner (2001) derive revenue equivalence under two different hypotheses. In the first, agents' type spaces must be convex and the valuation function of an agent is a convex function of the type of the agent. Under these conditions they show that every implementable rule satisfies revenue equivalence. The second hypothesis requires the allocation 
rule to satisfy certain differentiability and continuity conditions and the outcome space to be a subset of the Euclidean space. Furthermore, the valuation functions must be regular Lipschitzian and monotonically increasing in all arguments.

Milgrom and Segal (2002) show that revenue equivalence is a consequence of a particular envelope theorem in a setting where the type spaces are one-dimensional and the outcome space is arbitrary. An agent's valuation function is assumed differentiable and absolutely continuous in the type of the agent and the partial derivative of the valuation function with respect to the type must satisfy a certain integrability condition. Their result can be applied to multi-dimensional type spaces as well. In this case the type spaces must be smoothly connected and the valuation functions must be differentiable with bounded gradient.

We know of only two papers that identify necessary as well as sufficient conditions-i.e. characterizing conditions - for revenue equivalence to hold. If the outcome space is finite, Suijs (1996) characterizes type spaces and valuation functions for which utilitarian maximizers satisfy revenue equivalence. Chung and Olszewski (2007) characterize type spaces and valuation functions for which every implementable allocation rule satisfies revenue equivalence, again under the assumption of a finite outcome space. Furthermore, they derive sufficient conditions on the type spaces and valuation functions that generalize known results when the outcome space is countable or a probability distribution over a finite set of outcomes. In particular, they can show that some of the previously known conditions can be weakened for countable outcome spaces.

Our characterization differs from prior work in an important way. We identify a condition on the type spaces, the valuation functions and the implementable allocation rule together that characterize revenue equivalence. In other words, we prove that a particular allocation rule satisfies revenue equivalence if and only if this condition is satisfied. Our characterization differs from the one by Chung and Olszewski (2007) in three ways. First, ours holds for general outcome spaces. Second, our result implies revenue equivalence in cases where their result does not apply. In fact, given agents' type spaces and valuation functions, several allocation rules may be implementable in dominant strategies, some of which satisfy revenue equivalence and some do not. In this case, the conditions on the type space and valuation functions from their paper obviously cannot hold. However, our characterization can be used to determine which of the allocation rules do satisfy revenue equivalence. We give an example in Section 4.3. Third, the characterization in Chung and Olszewski (2007) is a 
corollary of our result, in the sense that their necessary and sufficient condition is naturally related to our graph theoretic interpretation of revenue equivalence. We refer to Section 4.2 for details. Moreover, our characterization yields elementary and direct alternative proofs for their sufficient conditions for countable infinite outcome spaces.

As in Chung and Olszewski (2007), a sufficient condition derived from the characterization yields a number of the earlier results as immediate consequences We list some of them below.

1. By restricting attention to countable outcome spaces we can relax the smooth connectedness condition on the type space invoked in Holmström (1979) to (topological) connectedness. In addition, our sufficient condition applies to any allocation that can be implemented in dominant strategies rather than just utilitarian maximizers.

2. The sufficient condition that Suijs (1996) derives from his characterization follows as a special case.

3. The sufficient condition of Krishna and Maenner (2001) under their first hypothesis when the outcome space is countable follows as a special case.

4. The sufficient condition of Milgrom and Segal (2002) when the outcome space is countable follows as a special case.

The remainder of the paper is organized as follows. In Section 2 we introduce notation and basic definitions. In Section 3, we derive our graph-theoretic characterization of revenue equivalence. In Section 4 we give three applications of the new characterization. First, a simple and elementary proof for a sufficient condition for revenue equivalence in a setting with countable outcome spaces. Second, we show how the characterization from Chung and Olszewski (2007) can be obtained as a consequence of ours. Finally, an example of an economic setting where our characterization can be used to identify revenue equivalence, whereas all known previous results are not applicable. We conclude with extensions to other notions of incentive compatibility in Section 5.

\section{Setting and Basic Concepts}

Denote by $\{1, \ldots, n\}$ the set of agents and let $A$ be the set of possible outcomes. Outcome space $A$ is allowed to have infinitely many, even uncountably many, elements. By $t_{i}$, we 
denote the type of agent $i \in\{1, \ldots, n\}$. Let $T_{i}$ be the type space of agent $i$. Type spaces $T_{i}$ can be arbitrary sets. Agent $i$ 's preferences over outcomes are modeled by the valuation function $v_{i}: A \times T_{i} \rightarrow \mathbb{R}$, where $v_{i}\left(a, t_{i}\right)$ is the valuation of agent $i$ for outcome $a$ when he has type $t_{i}$.

A mechanism $(f, \pi)$ consists of an allocation rule $f: \prod_{i=1}^{n} T_{i} \rightarrow A$ and a payment scheme $\pi: \prod_{i=1}^{n} T_{i} \rightarrow \mathbb{R}^{n}$. In a direct revelation mechanism, the allocation rule chooses for a vector $t$ of aggregate type reports of all agents an outcome $f(t)$, whereas the payment scheme assigns a payment $\pi_{i}(t)$ to each agent $i$. Let the vector $\left(t_{i}, t_{-i}\right)$ denote the aggregate type report vector when $i$ reports $t_{i}$ and the other agents' reports are represented by $t_{-i}$. We assume quasi-linear utilities, that is, the utility of agent $i$ when the aggregate report vector is $\left(t_{i}, t_{-i}\right)$ is $v_{i}\left(f\left(t_{i}, t_{-i}\right), t_{i}\right)-\pi_{i}\left(t_{i}, t_{-i}\right)$.

Definition 1 (dominant strategy incentive compatible) A direct revelation mechanism $(f, \pi)$ is called dominant strategy incentive compatible if for every agent $i$, every type $t_{i} \in T_{i}$, all aggregate type vectors $t_{-i}$ that the other agents could report and every type $s_{i} \in T_{i}$ that $i$ could report instead of $t_{i}$ :

$$
v_{i}\left(f\left(t_{i}, t_{-i}\right), t_{i}\right)-\pi_{i}\left(t_{i}, t_{-i}\right) \geq v_{i}\left(f\left(s_{i}, t_{-i}\right), t_{i}\right)-\pi_{i}\left(s_{i}, t_{-i}\right) .
$$

If for allocation rule $f$ there exists a payment scheme $\pi$ such that $(f, \pi)$ is a dominant strategy incentive compatible mechanism, then $f$ is called implementable in dominant strategies, in short implementable.

With the exception of Section 5, we assume that the allocation rule is implementable in dominant strategies and study the uniqueness of the corresponding payment scheme. We refer to the latter as revenue equivalence. ${ }^{4}$

Definition 2 (Revenue Equivalence) An allocation rule $f$ implementable in dominant strategies satisfies the revenue equivalence property if for any two dominant strategy incentive compatible mechanisms $(f, \pi)$ and $\left(f, \pi^{\prime}\right)$ and any agent $i$ there exists a function $h_{i}$ that only depends on the reported types of the other agents $t_{-i}$ such that

$$
\forall t_{i} \in T_{i}: \pi_{i}\left(t_{i}, t_{-i}\right)=\pi_{i}^{\prime}\left(t_{i}, t_{-i}\right)+h_{i}\left(t_{-i}\right) .
$$

\footnotetext{
${ }^{4}$ We choose the term revenue equivalence in accordance with Krishna (2002). It is equivalent to payoff equivalence as used in Krishna and Maenner (2001).
} 


\section{Characterization of Revenue Equivalence}

We give a necessary and sufficient condition for revenue equivalence with the aid of a graph theoretic interpretation used by Rochet (1987), Gui, Müller, and Vohra (2004) and Saks and $\mathrm{Yu}(2005)$ to characterize implementable allocation rules. We also adopt some of their notation.

Fix agent $i$ and let the reports of the other agents $t_{-i}$ be fixed as well. For simplicity of notation we write $T$ and $v$ instead of $T_{i}$ and $v_{i}$. Similarly, for any mechanism $(f, \pi)$, we regard $f$ and $\pi$ as functions of $i$ 's type alone, i.e. $f: T \rightarrow A$ and $\pi: T \rightarrow \mathbb{R}$. If $(f, \pi)$ is dominant strategy incentive compatible, it is easy to see that for any pair of types $s, t \in T$ such that $f(t)=f(s)=a$ for some $a \in A$, the payments must be equal, i.e. $\pi(t)=\pi(s)=: \pi_{a}$. Hence, the payment of agent $i$ is completely defined if the numbers $\pi_{a}$ are defined for all outcomes $a \in A$ such that $f^{-1}(a)$ is nonempty. For ease of notation, we let $A$ denote the set of outcomes that can be a achieved for some report of agent $i$. For an allocation rule $f$, let us define two different kinds of graphs. The type graph $T_{f}$ has node set $T$ and contains an arc from any node $s$ to any other node $t$ of length ${ }^{5}$

$$
\ell_{s t}=v(f(t), t)-v(f(s), t) .
$$

Here, $\ell_{s t}$ represents the gain in valuation for an agent truthfully reporting type $t$ instead of lying type $s$. This could be positive or negative. The allocation graph $G_{f}$ has node set $A$. Between any two nodes $a, b \in A$, there is a directed arc with length ${ }^{5}$

$$
\ell_{a b}=\inf _{t \in f^{-1}(b)}(v(b, t)-v(a, t))
$$

The arc lengths $\ell_{a b}$ in the allocation graph represent the least gain in valuation for any agent with type $t \in f^{-1}(b)$ for reporting truthfully, instead of misreporting so as to get outcome $a$ (instead of $b$ ). The type graph and allocation graph are complete, directed, and possibly infinite graphs ${ }^{6}$. We introduce our main results in terms of allocation graphs. Nevertheless, analogous results hold for type graphs as well.

\footnotetext{
${ }^{5}$ We assume that arc lengths are strictly larger than $-\infty$. For allocation rules implementable in dominant strategies this is no restriction, as the incentive compatibility constraints imply finiteness of the arc lengths.

${ }^{6}$ Clearly, type and allocation graph depend on the agent $i$ and reports $t_{-i}$ of the other agents. In order to keep notation simple, we will suppress this dependence on $i$ and $t_{-i}$ and will simply write $T_{f}$ and $G_{f}$.
} 
A path from node $a$ to node $b$ in $G_{f}$, or $(a, b)$-path for short, is defined as $P=(a=$ $\left.a_{0}, a_{1}, \ldots, a_{k}=b\right)$ such that $a_{i} \in A$ for $i=0, \ldots, k$. Denote by length $(P)$ the length of this path. A cycle is a path with $a=b$. For any $a$, we regard the path from $a$ to $a$ without any arcs as an $(a, a)$-path and define its length to be 0 . Define $\mathcal{P}(a, b)$ to be the set of all $(a, b)$-paths.

Definition 3 (Node Potential) A node potential $p$ is a function $p: A \rightarrow \mathbb{R}$ such that for all $x, y \in A, p(y) \leq p(x)+\ell_{x y}$.

Observation 1 Let $f$ be an allocation rule. Payment schemes $\pi$ such that $(f, \pi)$ is a dominant strategy incentive compatible mechanism, exactly correspond to node potentials in each of the allocation graphs $G_{f}$ that are obtained from a combination of an agent and a report vector of the other agents.

Proof. Assume $f$ is implementable. Fix agent $i$ and the reports $t_{-i}$ of the other agents. Consider the corresponding allocation graph $G_{f}$. For any pair of types $s, t \in T$ such that $f(t)=f(s)=a$ for some $a \in A$, the payments must be equal, i.e. $\pi(t)=\pi(s)=\pi_{a}$. Therefore, $\pi$ assigns a real number to every node in the graph. Incentive compatibility implies for any two outcomes $a, b \in A$ and all $t \in f^{-1}(b)$ that $v(b, t)-\pi_{b} \geq v(a, t)-\pi_{a}$, hence, $\pi_{b} \leq \pi_{a}+\ell_{a b}$.

For the other direction, define the payment $\pi$ for agent $i$ as follows. For any report vector of the other agents $t_{-i}$, consider the corresponding allocation graph $G_{f}$ and fix a node potential $p$. At aggregate report vector $\left(t_{i}, t_{-i}\right)$ with outcome $a=f\left(t_{i}, t_{-i}\right)$, let the payment be $\pi_{a}:=p(a)$. Incentive compatibility now follows from the fact that $p$ is a node potential in $G_{f}$, similarly to the above.

Let

$$
\operatorname{dist}_{G_{f}}(a, b)=\inf _{P \in \mathcal{P}(a, b)} \text { length }(P) .
$$

In general, $\operatorname{dist}_{G_{f}}(a, b)$ could be unbounded. However, if $G_{f}$ does not contain a negative cycle (the nonnegative cycle property), then $\operatorname{dist}_{G_{f}}(a, b)$ is finite, since the length of any $(a, b)$-path is lower bounded by $-\ell_{b a}$.

Lemma 1 Fix an agent and some report vector of the other agents. The corresponding allocation graph $G_{f}$ has a node potential if and only if it satisfies the nonnegative cycle property. 
Proof. Proofs can be found e.g. in Schrijver (2003) for finite $A$ and in Rochet (1987) for infinite $A$. For the sake of completeness, we give a simple proof here. If $G_{f}$ has no negative cycle, then for any $a \in A$, $\operatorname{dist}_{G_{f}}(a, \cdot)$ is well-defined, i.e. takes only finite values. The distances $\operatorname{dist}_{G_{f}}(a, \cdot)$ define a node potential, because $\operatorname{dist}_{G_{f}}(a, x) \leq \operatorname{dist}_{G_{f}}(a, y)+\ell_{y x}$ for all $x, y \in A$. On the other hand, given a node potential $p$, add up the inequalities $p(y)-p(x) \leq \ell_{x y}$ for all arcs $(x, y)$ on a cycle to prove that the cycle has nonnegative length.

Observation 1 together with Lemma 1 yields a characterization of allocation rules that are implementable in dominant strategies (see also e.g. Rochet (1987)).

Observation 2 The allocation rule $f$ is implementable in dominant strategies if and only if all allocation graphs $G_{f}$ obtained from a combination of an agent and a report vector of the other agents satisfy the nonnegative cycle property.

From Lemma 1 and observations 1 and 2 it follows that for any allocation rule $f$ implementable in dominant strategies, there exist node potentials in all allocation graphs $G_{f}$. The allocation rule $f$ satisfies revenue equivalence if and only if in each allocation graph $G_{f}$, the node potential is uniquely defined up to a constant. Our main result states that this is the case if and only if distances are anti-symmetric in every $G_{f}$.

Theorem 1 (Characterization of Revenue Equivalence) Let $f$ be an allocation rule that is implementable in dominant strategies. $f$ satisfies revenue equivalence if and only if in all allocation graphs $G_{f}$ obtained from a combination of an agent and a report vector of the other agents, distances are anti-symmetric, i.e. dist $_{G_{f}}(a, b)=-\operatorname{dist}_{G_{f}}(b, a)$ for all $a, b \in A$.

Proof. Suppose first that $f$ satisfies revenue equivalence. Fix a combination of an agent and a report vector of the other agents and let $G_{f}$ be the corresponding allocation graph. Let $a, b \in A$. The functions $\operatorname{dist}_{G_{f}}(a, \cdot)$ and $\operatorname{dist}_{G_{f}}(b, \cdot)$ are node potentials in $G_{f}$. As any two node potentials differ only by a constant, we have that $\operatorname{dist}_{G_{f}}(a, \cdot)-\operatorname{dist}_{G_{f}}(b, \cdot)$ is a constant function. Especially, for $a$ and $b$ we get that $\operatorname{dist}_{G_{f}}(a, a)-\operatorname{dist}_{G_{f}}(b, a)=\operatorname{dist}_{G_{f}}(a, b)-$ $\operatorname{dist}_{G_{f}}(b, b)$. Clearly, $\operatorname{dist}_{G_{f}}(a, a)=\operatorname{dist}_{G_{f}}(b, b)=0$ and hence $\operatorname{dist}_{G_{f}}(a, b)=-\operatorname{dist}_{G_{f}}(b, a)$.

Now suppose that $\operatorname{dist}_{G_{f}}(a, b)=-\operatorname{dist}_{G_{f}}(b, a)$ for all $a, b \in A$. Let $a, b \in A$. Let $P_{a b}$ be an $(a, b)$-path with nodes $a=a_{0}, a_{1}, \ldots, a_{k}=b$. For any node potential $p$ add up the 
inequalities $p\left(a_{i}\right)-p\left(a_{i-1}\right) \leq \ell_{a_{i-1} a_{i}}$ for $i=1, \ldots, k$. This yields $p(b)-p(a) \leq$ length $\left(P_{a b}\right)$. Therefore,

$$
p(b)-p(a) \leq \inf _{P \in \mathcal{P}(a, b)} \text { length }(P)=\operatorname{dist}_{G_{f}}(a, b) .
$$

Similarly, $p(a)-p(b) \leq \operatorname{dist}_{G_{f}}(b, a)$. Therefore, $-\operatorname{dist}_{G_{f}}(b, a) \leq p(b)-p(a) \leq \operatorname{dist}_{G_{f}}(a, b)$. Since $\operatorname{dist}_{G_{f}}(a, b)=-\operatorname{dist}_{G_{f}}(b, a), p(b)-p(a)=\operatorname{dist}_{G_{f}}(a, b)$ for any node potential $p$. Hence, any potential is completely defined, once $p(a)$ has been chosen for some outcome $a$. Thus, any two node potentials can only differ by a constant and $f$ satisfies revenue equivalence.

An analogous characterization holds for type graphs as well. One can check that all previous arguments still apply when using type graphs. On the other hand, note the following relation of node potentials in $G_{f}$ and node potentials in $T_{f}$. Given a node potential $p^{G}$ in $G_{f}$, we can define a node potential $p^{T}$ in $T_{f}$ by letting $p^{T}(t):=p^{G}(f(t))$ for any $t \in T$. In fact, let $\ell^{G}$ and $\ell^{T}$ denote the arc lengths in $G_{f}$ and $T_{f}$ respectively and observe that $\ell_{a b}^{G}=\inf \left\{\ell_{s t}^{T} \mid s \in f^{-1}(a), t \in f^{-1}(b)\right\}$. Then, for any $s, t \in T, p^{T}(t)=p^{G}(f(t)) \leq$ $p^{G}(f(s))+\ell_{f(s) f(t)}^{G} \leq p^{T}(s)+\ell_{s t}^{T}$ and $p^{T}$ is a node potential. On the other hand, given a node potential $p^{T}$ in $T_{f}$, let $p^{G}(a):=p^{T}(s)$ for any $s \in f^{-1}(a)$. Note that $p^{G}$ is welldefined as $f(s)=f(t)=a$ implies $\ell_{s t}^{T}=0$ and hence $p^{T}(s)=p^{T}(t)$. Furthermore, for any $a, b \in A$ and any $s \in f^{-1}(a), t \in f^{-1}(b), p^{G}(a)=p^{T}(s) \leq p^{T}(t)+\ell_{t s}^{T}=p^{G}(b)+\ell_{t s}^{T}$. Hence, $p^{G}(a) \leq p^{G}(b)+\ell_{b a}^{G}$ and $p^{G}$ is a node potential in $G_{f}$. Consequently, there is a one-to-one relationship between node potentials in $G_{f}$ and node potentials in $T_{f}$. This insight together with a proof similar to the one of Theorem 1 yield the following corollary.

Corollary 1 (Characterization of Revenue Equivalence on Type Graphs) Let $f$ be an allocation rule that is implementable in dominant strategies. Then $f$ satisfies revenue equivalence if and only if in all type graphs $T_{f}$ obtained from a combination of an agent and a report vector of the other agents, distances are anti-symmetric, i.e. dist $_{T_{f}}(s, t)=$ - dist $_{T_{f}}(t, s)$ for all $s, t \in T$.

\section{Applications}

In this section we give three applications of our main result. The first shows how Theorem 1 yields a simple transparent proof that all implementable $f$ satisfy revenue equivalence in a 
setting where the outcome space is countable. The second shows that the characterization of Chung and Olszewski (2007) can be derived from Theorem 1. The third describes an economic environment with uncountable outcome space where Theorem 1 can be used to identify revenue equivalence, but where the existing theorems are silent.

\subsection{Countable Outcome Spaces}

We investigate the case of countably infinite outcome spaces in this section. Theorem 1 yields an elementary proof for revenue equivalence under weak assumptions.

Theorem 2 Let $A$ be a countable outcome space. Let each agent $i \in\{1, \ldots, n\}$ have types from the (topologically) connected type space $T_{i} \subseteq \mathbb{R}^{k_{i}}$. Let the family of valuation functions $\left\{v_{i}(a, \cdot)\right\}_{a \in A}$ be equicontinuous ${ }^{7}$ for every agent $i$. Then, every allocation rule $f: \Pi_{i=1}^{n} T_{i} \rightarrow A$ implementable in dominant strategies satisfies revenue equivalence.

Proof. Assume an allocation rule $f$ implementable in dominant strategies that does not satisfy revenue equivalence. We show this implies that there is an agent $i$ whose type space is not connected. According to Theorem 1, there is an agent $i$ and a report vector of the other agents $t_{-i}$ with corresponding allocation graph $G_{f}$ and $a^{*}, b^{*} \in A$ such that $\operatorname{dist}_{G_{f}}\left(a^{*}, b^{*}\right)+$ $\operatorname{dist}_{G_{f}}\left(b^{*}, a^{*}\right)>0$. Let $T$ be the type space of $i$ and regard $f$ as a function on $T$ as before. As $A$ is countable, the set $\left\{\operatorname{dist}_{G_{f}}\left(a^{*}, x\right)+\operatorname{dist}_{G_{f}}\left(x, a^{*}\right) \mid x \in A\right\}$ contains only countably many values. Hence, there exists a $z>0$ such that the sets $A_{1}=\left\{x \mid \operatorname{dist}_{G_{f}}\left(a^{*}, x\right)+\operatorname{dist}_{G_{f}}\left(x, a^{*}\right)<\right.$ $z\}$ and $A_{2}=\left\{x \mid\right.$ dist $_{G_{f}}\left(a^{*}, x\right)+$ dist $\left._{G_{f}}\left(x, a^{*}\right)>z\right\}$ are both non-empty and together yield a partition of $A$. Then, the sets $T_{1}=f^{-1}\left(A_{1}\right)$ and $T_{2}=f^{-1}\left(A_{2}\right)$ are non-empty and yield a partition of the type space $T$. $T_{1}$ is a proper subset of $T$. We show that $T_{1}$ is open and closed, implying that $T$ is not connected.

$T_{1}$ is open: Let $t \in T_{1}$. Let $f(t)=x \in A_{1}$. Then, $\operatorname{dist}_{G_{f}}\left(a^{*}, x\right)+\operatorname{dist}_{G_{f}}\left(x, a^{*}\right)=z-\varepsilon$ for some $\varepsilon=\varepsilon(x)>0$. As the $v(a, \cdot), a \in A$, are equicontinuous, there is a $\delta_{\varepsilon / 2}$ such that $|v(a, t)-v(a, s)|<\varepsilon / 2$ for all $a \in A$ and $s \in T$ with $\|s-t\|<\delta_{\varepsilon / 2}$. Let $s \in T$ such that

\footnotetext{
${ }^{7}\{v(a, \cdot)\}_{a \in A}$ is equicontinuous if for all $\varepsilon>0$ and $t \in T \subseteq \mathbb{R}^{k}$ there is $\delta>0$ such that for all outcomes $a \in A$ and all $s \in T$ with $\|t-s\|<\delta$ it holds that $|v(a, t)-v(a, s)|<\varepsilon$. Especially, $\delta$ may not depend on $a$. $\|\cdot\|$ denotes the Euclidean norm on $\mathbb{R}^{k}$.
} 
$\|s-t\|<\delta_{\varepsilon / 2}$ and let $y=f(s)$. Then the following is true:

$$
\begin{aligned}
\operatorname{dist}_{G_{f}}\left(a^{*}, y\right)+\operatorname{dist}_{G_{f}}\left(y, a^{*}\right) & \leq \operatorname{dist}_{G_{f}}\left(a^{*}, x\right)+\ell_{x y}+\ell_{y x}+\operatorname{dist}_{G_{f}}\left(x, a^{*}\right) \\
& =z-\varepsilon+\ell_{x y}+\ell_{y x} \\
& \leq z-\varepsilon+|v(y, s)-v(y, t)|+|v(x, t)-v(x, s)| \\
& <z
\end{aligned}
$$

Hence $\operatorname{dist}_{G_{f}}\left(a^{*}, y\right)+\operatorname{dist}_{G_{f}}\left(y, a^{*}\right)<z$. Thus $y \in A_{1}$ and $s \in T_{1}$ for any $s$ in the $\delta_{\varepsilon / 2}$-ball around $t$. Consequently, $T_{1}$ is open.

$T_{1}$ is closed: Let $\left(t^{n}\right)_{n \in \mathbb{N}}$ be a sequence in $T_{1}$ that converges to $t \in T$. Suppose for contradiction that $t \in T_{2}$. Let $x=f(t)$. Then $\operatorname{dist}_{G_{f}}\left(a^{*}, x\right)+\operatorname{dist}_{G_{f}}\left(x, a^{*}\right)=z+\varepsilon$ for some $\varepsilon=\varepsilon(x)>0$. By equicontinuity, there is a $\delta_{\varepsilon / 2}$ such that $|v(a, t)-v(a, s)|<\varepsilon / 2$ for all $a \in A$ and $s \in T$ with $\|s-t\|<\delta_{\varepsilon / 2}$. Choose $n_{0}$ such that $\left\|t^{n_{0}}-t\right\|<\delta_{\varepsilon / 2}$. Let $y=f\left(t^{n_{0}}\right)$. As $t^{n_{0}} \in T_{1}, \operatorname{dist}_{G_{f}}\left(a^{*}, y\right)+\operatorname{dist}_{G_{f}}\left(y, a^{*}\right)<z$. Then the following holds:

$$
\begin{aligned}
z+\varepsilon & =\operatorname{dist}_{G_{f}}\left(a^{*}, x\right)+\operatorname{dist}_{G_{f}}\left(x, a^{*}\right) \\
& \leq \operatorname{dist}_{G_{f}}\left(a^{*}, y\right)+\ell_{y x}+\ell_{x y}+\operatorname{dist}_{G_{f}}\left(y, a^{*}\right) \\
& <z+\ell_{y x}+\ell_{x y} \\
& \leq z+\left|v(x, t)-v\left(x, t^{n_{0}}\right)\right|+\left|v\left(y, t^{n_{0}}\right)-v(y, t)\right| \\
& <z+\varepsilon
\end{aligned}
$$

a contradiction.

An example in Section 4.3 demonstrates that Theorem 2 cannot be generalized to uncountable outcome spaces.

Since equicontinuity of $\left\{v_{i}(a, \cdot)\right\}_{a \in A}$ for finite $A$ is equivalent to the requirement that $v_{i}(a, \cdot)$ be continuous for all $a \in A$ we obtain the following Corollary for the case $|A|$ finite.

Corollary 2 Let $A$ be a finite outcome space. Let each agent $i \in\{1, \ldots, n\}$ have types from the (topologically) connected type space $T_{i} \subseteq \mathbb{R}^{k_{i}}$. Let each agent's valuation function $v_{i}(a, \cdot)$ be a continuous function in the type of the agent for every outcome $a \in A$. Then, every allocation rule $f: \prod_{i=1}^{n} T_{i} \rightarrow A$ that is implementable in dominant strategies satisfies revenue equivalence. 
A different way of modeling valuations for countable outcome spaces is to identify types with vectors in $\mathbb{R}^{A}$, such that $v_{i}(a, t)=t_{a}$. On $\mathbb{R}^{A}$ we can define the sup-topology by defining for $\varepsilon>0$, and $t \in \mathbb{R}^{A}$ the $\varepsilon$-ball around $t$ as $B_{\varepsilon}(t)=\left\{s \in \mathbb{R}^{A}\left|\sup _{a \in A}\right| t_{a}-s_{a} \mid<\varepsilon\right\}$. Using almost the same proof as for Theorem 2, one can show the following theorem, which can also be found in Chung and Olszewski (2007).

Theorem 3 Let $A$ be a countable outcome space. Let the type space $T_{i} \subseteq \mathbb{R}^{A}$ for every agent $i$ be (topologically) connected with respect to the sup-topology on $\mathbb{R}^{A}$. Then, every allocation rule $f: \prod_{i=1}^{n} T_{i} \rightarrow A$ that is implementable also satisfies revenue equivalence.

\subsection{The Characterization by Chung and Olszewski}

In this section, we show how the characterization by Chung and Olszewski (2007) follows from our main result and comment briefly on the results in other papers on revenue equivalence. First, we introduce the notation used by Chung and Olszewski (2007) and restate their characterization theorem.

Let $A$ be countable. As before, regard everything from the perspective of a single agent. Let $A_{1}, A_{2}$ be disjoint subsets of $A$ and $r: A_{1} \cup A_{2} \rightarrow \mathbb{R}$. For every $\varepsilon>0$, let

$$
\mathcal{T}_{1}(\varepsilon)=\bigcup_{a_{1} \in A_{1}}\left\{t \in T \mid \forall a_{2} \in A_{2}: v\left(a_{1}, t\right)-v\left(a_{2}, t\right)>r\left(a_{1}\right)-r\left(a_{2}\right)+\varepsilon\right\}
$$

and

$$
\mathcal{T}_{2}(\varepsilon)=\bigcup_{a_{2} \in A_{2}}\left\{t \in T \mid \forall a_{1} \in A_{1}: v\left(a_{1}, t\right)-v\left(a_{2}, t\right)<r\left(a_{1}\right)-r\left(a_{2}\right)-\varepsilon\right\} .
$$

Finally, let $\mathcal{T}_{i}=\cup_{\varepsilon>0} \mathcal{T}_{i}(\varepsilon), i=1,2$. Observe that $\mathcal{T}_{1} \cap \mathcal{T}_{2}=\emptyset$. Call the type space $T$ splittable if there are $A_{1}, A_{2}$ and $r$ such that $T=\mathcal{T}_{1} \cup \mathcal{T}_{2}$ and $\mathcal{T}_{i} \neq \emptyset$ for $i=1,2$. Note that, if $T$ is splittable, $T$ is disconnected with respect to the sup-topology on $\mathbb{R}^{A}$ defined above, as $\mathcal{T}_{1}$ and $\mathcal{T}_{2}$ are open sets. $T$ being topologically disconnected, does not imply that $T$ is splittable.

Theorem 4 (Chung and Olszewski 2007) If $A$ is finite then the following two statements are equivalent.

(i) All $f$ that are implementable in dominant strategies satisfy revenue equivalence.

(ii) For all agents $T_{i}$ is not splittable. 
If $A$ is not finite, but countable, (ii) implies ( $i)$.

Notice that in Theorem 1 no assumption on the cardinality of $A$ is made, whereas in Theorem 4, $A$ is assumed finite or countable, respectively. On the other hand, Theorem 1 imposes a condition on the allocation rule, whereas Theorem 4 characterizes $T$ and $v$ such that all allocation rules that are implementable in dominant strategies satisfy revenue equivalence. We elaborate on this difference in Section 4.3.

In order to show that $(i i)$ in Theorem 4 is a necessary condition for revenue equivalence in the case of finite $A$, one can directly construct an allocation rule and two payment schemes that do not differ by a constant from the assumption that $T$ is splittable. This is done in the paper by Chung and Olszewski. We give an alternative proof for the fact that (ii) is a sufficient condition for countable $A$. Our proof establishes a connection to the allocation graph defined in Section 3.

Proof. $[(i i) \Rightarrow(i)]$ Suppose an allocation rule $f$ that is implementable in dominant strategies but does not satisfy revenue equivalence. Since $f$ is implementable, the allocation graphs satisfy the non-negative cycle property. Since revenue equivalence is violated, Theorem 1 implies that there is an agent $i$ and reports of the other agents $t_{-i}$ such that in the corresponding allocation graph $G_{f}$, dist $G_{f}\left(a^{*}, b^{*}\right)+\operatorname{dist}_{G_{f}}\left(b^{*}, a^{*}\right)>0$ for some $a^{*}, b^{*} \in A$. Assume the the perspective of agent $i$. In the following, we write $\operatorname{dist}(\cdot, \cdot) \operatorname{instead}$ of $\operatorname{dist}_{G_{f}}(\cdot, \cdot)$ and $T$ instead of $T_{i}$ for ease of notation. We show the above assumptions imply that $T$ is splittable.

Define $d(a)=\operatorname{dist}\left(a^{*}, a\right)+\operatorname{dist}\left(a, a^{*}\right)$ for all $a \in A$. Since the function $d$ takes only countably many values, there exists $z \in \mathbb{R}$ such that the following sets form a non-trivial partition of $A: A_{1}=\{a \in A \mid d(a)>z\}, A_{2}=\{a \in A \mid d(a)<z\}$. Observe that for every $a_{1} \in A_{1}$, there exists $\varepsilon\left(a_{1}\right)>0$ such that $d\left(a_{1}\right)>z+\varepsilon\left(a_{1}\right)$. Similarly, for every $a_{2} \in A_{2}$, there exists $\varepsilon\left(a_{2}\right)>0$ such that $d\left(a_{2}\right)<z-\varepsilon\left(a_{2}\right)$.

For $a_{1} \in A_{1}$, let $r\left(a_{1}\right)=-\operatorname{dist}\left(a_{1}, a^{*}\right)$. For $a_{2} \in A_{2}$, let $r\left(a_{2}\right)=\operatorname{dist}\left(a^{*}, a_{2}\right)-z$. Now let $t \in T$ such that $f(t)=a_{1} \in A_{1}$. We claim that for all $a_{2} \in A_{2}$ it holds that $v\left(a_{1}, t\right)-v\left(a_{2}, t\right)>$ $r\left(a_{1}\right)-r\left(a_{2}\right)+\varepsilon\left(a_{1}\right)$, which proves $t \in \mathcal{T}_{1}\left(\varepsilon\left(a_{1}\right)\right)$. Indeed, $v\left(a_{1}, t\right)-v\left(a_{2}, t\right) \geq \ell\left(a_{2}, a_{1}\right)$. The claim then follows from:

$$
\begin{aligned}
\operatorname{dist}\left(a^{*}, a_{2}\right)+\ell\left(a_{2}, a_{1}\right)+\operatorname{dist}\left(a_{1}, a^{*}\right) & \geq \operatorname{dist}\left(a^{*}, a_{1}\right)+\operatorname{dist}\left(a_{1}, a^{*}\right) \\
& >z+\varepsilon\left(a_{1}\right) .
\end{aligned}
$$


Next, let $t \in T$ such that $f(t)=a_{2} \in A_{2}$. We claim that for all $a_{1} \in A_{1}$ it holds that $v\left(a_{1}, t\right)-v\left(a_{2}, t\right)<r\left(a_{1}\right)-r\left(a_{2}\right)-\varepsilon\left(a_{2}\right)$, which proves $t \in \mathcal{T}_{2}\left(\varepsilon\left(a_{2}\right)\right)$. Again, $v\left(a_{1}, t\right)-v\left(a_{2}, t\right) \leq$ $-\ell\left(a_{1}, a_{2}\right)$, and the claim follows from:

$$
\begin{aligned}
& \operatorname{dist}\left(a^{*}, a_{2}\right)+\operatorname{dist}\left(a_{1}, a^{*}\right)-\ell\left(a_{1}, a_{2}\right) \\
\leq & \operatorname{dist}\left(a^{*}, a_{2}\right)+\operatorname{dist}\left(a_{1}, a^{*}\right)-\operatorname{dist}\left(a_{1}, a^{*}\right)+\operatorname{dist}\left(a_{2}, a^{*}\right) \\
= & \operatorname{dist}\left(a^{*}, a_{2}\right)+\operatorname{dist}\left(a_{2}, a^{*}\right) \\
< & z-\varepsilon\left(a_{2}\right) .
\end{aligned}
$$

Using Theorem 4, Chung and Olszewski derive Theorem 3 for the case of countable outcome spaces and show how the results of prior work mentioned in the introduction (Green and Laffont 1977, Holmström 1979, Krishna and Maenner 2001, Milgrom and Segal 2002) follow from that theorem. In a similar way, those results are implied by Theorem 2. We therefore refer to Chung and Olszewski (2007) for a detailed discussion of the mentioned literature. Furthermore, the sufficient condition that Suijs (1996) derives from his characterization follows easily from Corollary 2 and the fact that path-connectedness implies connectedness.

\subsection{A Setting with an Uncountable Outcome Space}

In this section, we give an example for an economic setting where Theorem 1 can be used to identify revenue equivalence, where all previous results are not applicable.

Cachon and Lariviere (1999) consider demand rationing problems where agents have to share a divisible good. We consider the following variant of the problem. A supplier has one unit of a perfectly divisible good that has to be distributed among $n$ retailers (agents). The type of agent $i$ is his demand $t_{i} \in(0,1]$. Given the reports $t \in(0,1]^{n}$ of all agents, an allocation rule $f:(0,1]^{n} \rightarrow[0,1]^{n}$ assigns a fraction of the good to every agent such that $\sum_{i=1}^{n} f_{i}(t) \leq 1$. If an agent's demand is met, he incurs a disutility of 0 , otherwise his disutility is linear in the amount of unmet demand. More precisely, agent $i$ 's valuation ${ }^{8}$ if he is assigned

\footnotetext{
${ }^{8}$ This valuation function appears in Holmström (1979) as an example to demonstrate that his smooth path-connectedness assumption cannot be weakened. Likewise, the example can be used to show that the convexity assumption of the valuation function in Krishna and Maenner (2001) cannot be relaxed.
} 
quantity $q_{i}$ is

$$
v_{i}\left(q_{i}, t_{i}\right)= \begin{cases}0, & \text { if } q_{i} \geq t_{i}, \\ q_{i}-t_{i}, & \text { if } q_{i}<t_{i} .\end{cases}
$$

In this context, payments are reimbursements by the supplier for unmet demand.

Let us call an allocation rule $f$ dictatorial, if there is an agent $i$ that always gets precisely his demanded quantity, $f_{i}\left(t_{i}, t_{-i}\right)=t_{i}$ for all $t_{-i}$. We show that any dictatorial rule violates revenue equivalence.

Theorem 5 For the above demand rationing problem, let $f_{1}(t)=t_{1}$ and $f_{i}(t)=\left(1-t_{1}\right) /(n-$ 1). Then $f$ is implementable but does not satisfy revenue equivalence.

Theorem 5 is formulated for the allocation rule that splits the remaining supply equally among agents 2 to $n$. However, from the proof it can be easily seen that the conclusion of Theorem 5 holds for all other dictatorial rules that are implementable.

Proof. [Theorem 5] Note that for agents $2, \ldots, n$ their assigned quantity does not depend on their report. Therefore, truthful reporting is a (weakly) dominant strategy for those agents. Regard the type graph for agent 1 and note that it does not depend on the report of the other agents. For simplicity we use $v, t$ and $f$ instead of $v_{1}, t_{1}$ and $f_{1}$. Consider the type graph $T_{f}$. Let $s, t \in(0,1]$ with $s<t$. We call $(s, t)$ a forward arc and $(t, s)$ a backward arc. Then

$$
\begin{aligned}
& \ell_{s t}=v(f(t), t)-v(f(s), t)=v(t, t)-v(s, t)=t-s>0, \text { and } \\
& \ell_{t s}=v(f(s), s)-v(f(t), s)=v(s, s)-v(t, s)=0 .
\end{aligned}
$$

As all arcs have non-negative length, there is no negative cycle and $f$ is implementable. Furthermore, $\operatorname{dist}_{T_{f}}(t, s)=\ell_{t s}=0$ for $t>s$. We claim that $\operatorname{dist}_{T_{f}}(s, t)=t-s>0$. To that end, note that all paths from $s$ to $t$ that use only forward arcs have the same length $t-s$. If a path from $s$ to $t$ contains a backward arc, the forward arcs of that path have a total length more than $t-s$. Hence, for all $s<t$ we have $d_{i s t_{T_{f}}}(s, t)+d i s t_{T_{f}}(t, s)>0$. Therefore revenue equivalence does not hold according to Theorem 1 . In fact, $\pi_{1}(t)=0$ for all $t \in(0,1]$ and $\pi_{1}(t)=t-1$ for all $t \in(0,1]$ are two payment schemes for agent 1 that make $f$ dominant strategy incentive compatible. (For the other agents, pick any constant payment scheme.) 
Theorem 5 implies that in this setting not all implementable $f$ satisfy revenue equivalence. A theorem describing sufficient conditions for all implementable $f$ to satisfy revenue equivalence is necessarily silent here. Nevertheless, we can use Theorem 1 to identify properties of allocation rules that guarantee revenue equivalence in this setting. We state such properties formally in Theorem 6 below, and then show that for instance the proportional allocation rule with $f_{i}(t)=t_{i} / \sum_{j=1}^{n} t_{j}$ satisfies these properties.

Theorem 6 Let $f:(0,1]^{n} \rightarrow[0,1]^{n}$ be an allocation rule. If for every agent $i$ and every report $t_{-i}$ of the other agents, $f$ satisfies any of the conditions below, then $f$ is implementable and satisfies revenue equivalence.

(i) $f_{i}\left(t_{i}, t_{-i}\right)$ is continuous in $t_{i}$ and $f_{i}\left(t_{i}, t_{-i}\right)<t_{i}$ for all $t_{i} \in(0,1]$,

(ii) $f_{i}\left(t_{i}, t_{-i}\right)$ is continuous in $t_{i}$ and $f_{i}\left(t_{i}, t_{-i}\right)>t_{i}$ for all $t_{i} \in(0,1), f_{i}\left(1, t_{-i}\right)=1$,

(iii) $f_{i}\left(t_{i}, t_{-i}\right)$ is continuous and increasing in $t_{i}$ and has exactly one fixed point $x \in(0,1]$ with $f_{i}\left(x, t_{-i}\right)=x, f_{i}\left(t_{i}, t_{-i}\right)>t_{i}$ for all $t_{i} \in(0, x), f_{i}\left(t_{i}, t_{-i}\right)<t_{i}$ for all $t_{i} \in(x, 1]$.

Proof. Again, fix agent $i, t_{-i}$ and use $v, t$ and $f$ instead of $v_{i}, t_{i}$ and $f_{i}$. Furthermore, regard $f$ as a function of $i$ 's type only. For all three cases, assume that $f$ is continuous. Regard the corresponding type graph $T_{f}$.

(i) We have $f(t)<t$ for $t \in(0,1]$. Let $s<t$. Using $f(s)<s<t$, the arc lengths in $T_{f}$ are as follows:

$$
\begin{aligned}
& \ell_{s t}=f(t)-f(s) \text { and } \\
& \ell_{t s}= \begin{cases}f(s)-f(t), & \text { if } f(t)<s, \\
f(s)-s, & \text { if } f(t) \geq s .\end{cases}
\end{aligned}
$$

To verify implementability, we prove that any cycle in the type graph has non-negative length. Note that for $s<t, \ell_{s t}=\ell_{s x}+\ell_{x t}$ for any $s<x<t$. We say that we split arc $(s, t)$ at $x$ if we replace $(s, t)$ by $\operatorname{arcs}(s, x)$ and $(x, t)$ in a path or cycle. Note that splitting forward arcs does not change the length of the path or cycle. Consider any finite cycle $c$ with nodes $c_{1}$ to $c_{k}$ and rename the nodes such that $c_{1}<c_{2}<\cdots<c_{k}$. Split every forward arc $\left(c_{u}, c_{v}\right)$ with $u<v$ at all intermediate nodes $c_{u+1}, \ldots, c_{v-1}$ and call the resulting cycle $c^{\prime}$; the cycle length remains the same. Consider some backward arc $\left(c_{v}, c_{u}\right)$ with $u<v$. As $c^{\prime}$ is a cycle, it contains all 
forward $\operatorname{arcs}\left(c_{u}, c_{u+1}\right), \ldots,\left(c_{v-1}, c_{v}\right)$. The length of the sub-cycle $\left(c_{u}, c_{u+1}, \ldots, c_{v-1}, c_{v}, c_{u}\right)$ is equal to

$$
\ell_{c_{u} c_{u+1}}+\ldots+\ell_{c_{v-1} c_{v}}+\ell_{c_{v} c_{u}}=\ell_{c_{u} c_{v}}+\ell_{c_{v} c_{u}}= \begin{cases}f\left(c_{v}\right)-c_{u}, & \text { if } f\left(c_{v}\right) \geq c_{u} \\ 0, & \text { if } c_{u}>f\left(c_{v}\right)\end{cases}
$$

and hence is non-negative. Removing the arcs of the sub-cycle $\left(c_{u}, c_{u+1}, \ldots, c_{v-1}, c_{v}, c_{u}\right)$ from $c^{\prime}$ leaves a new cycle $c^{\prime \prime}$ of smaller or equal length. As $c^{\prime \prime}$ is still a cycle we can repeat the argument finitely many times and finally arrive at the empty cycle with length 0 . Hence, $c$ has non-negative length and $f$ is implementable.

In order to verify that $f$ satisfies revenue equivalence, we compute distances $\operatorname{dist}_{T_{f}}(s, t)$ and $\operatorname{dist}_{T_{f}}(t, s)$ for $s<t$. Note that $\operatorname{dist}_{T_{f}}(s, t) \leq \ell_{s t}=f(t)-f(s)$. We claim that also $d_{i s t_{T_{f}}}(t, s) \leq f(s)-f(t)$, so $d_{i s t_{T_{f}}}(s, t)+d i s t_{T_{f}}(t, s) \leq 0$. Together with implementability we get $\operatorname{dist}_{T_{f}}(s, t)+\operatorname{dist}_{T_{f}}(t, s)=0$, and revenue equivalence holds by Corollary 1 . To prove the claim we consider two cases. If $f(t)<s$, dist $T_{f}(t, s) \leq \ell_{t s}=f(s)-f(t)$ and we are done. If $f(t) \geq s$, consider the sequence $\left(x_{n}\right)_{n=0}^{\infty}=(t, f(t), f(f(t)), \ldots)$. It is monotonically decreasing and bounded and so converges. The sequence $\left(f\left(x_{n}\right)\right)_{n=0}^{\infty}=\left(f(t), f(f(t)), f^{3}(t), \ldots\right)$ converges for the same reason and $\lim _{n \rightarrow \infty} x_{n}=\lim _{n \rightarrow \infty} f\left(x_{n}\right)=: x$. As $f$ is continuous, we have $x=f(x)$ if $x$ is in $(0,1]$. As there is no fixed point in $(0,1]$, we conclude $x=0$. Therefore, there exists some smallest index $K$ such that $x_{K}<s$. Now consider the path $P_{K}$ defined as $\left(t=x_{0}, \ldots, x_{K}, s\right)$. Path $P_{K}$ has length $f^{2}(t)-f(t)+f^{3}(t)-f^{2}(t)+\cdots+f^{K+1}(t)-$ $f^{K}(t)+f(s)-f^{K+1}(t)=f(s)-f(t)$. We conclude $\operatorname{dist}_{T_{f}}(t, s) \leq \ell\left(P_{K}\right)=f(s)-f(t)$, and we are done.

(ii) We have $f(t)>t$ for $t \in(0,1)$ and $f(1)=1$. Let $s<t$. Using $f(t) \geq t>s$, the arc lengths in $T_{f}$ can be computed as follows:

$$
\begin{aligned}
& \ell_{s t}=\left\{\begin{array}{ll}
0, & \text { if } f(s) \geq t ; \\
t-f(s), & \text { if } f(s)<t
\end{array}\right. \text { and } \\
& \ell_{t s}=0
\end{aligned}
$$

As all arc lengths are non-negative, all cycles have non-negative length and $f$ is implementable.

Since all paths have non-negative length, we know that $\operatorname{dist}_{T_{f}}(t, s)=\ell_{t s}=0$. We 
claim that also $\operatorname{dist}_{T_{f}}(s, t)=0$, hence revenue equivalence holds by Corollary 1. If $f(s) \geq$ $t$, dist $T_{f}(s, t)=\ell_{s t}=0$ and we are done. If $f(s)<t$, regard the sequence $\left(x_{n}\right)_{n=0}^{\infty}=$ $(s, f(s), f(f(s)), \ldots)$. This sequence converges to fixed point $x=1$ by the same arguments as in $(i)$. Let us regard paths $P_{k}$ defined as $P_{k}=\left(s=x_{0}, \ldots, x_{k}, 1\right)$. As $\ell_{x_{i}, f\left(x_{i}\right)}=0$ for $i=0, \ldots, k-1$ and $\ell_{x_{k}, 1}=1-f\left(x_{k}\right)$, the length of $P_{k}$ is $1-f\left(x_{k}\right)$, which converges to 0 as $k$ increases. Thus, $\operatorname{dist}_{T_{f}}(s, 1)=0$. With $\ell_{1 t}=0$ it follows that $\operatorname{dist}_{T_{f}}(s, t)=0$.

(iii) We have that $f$ is increasing on $(0,1]$ and there is $x \in(0,1)$ such that $f(x)=x$, $f(t)>t$ for $t \in(0, x)$, and $f(t)<t$ for $t \in(x, 1]$. There are six types of arcs, whose lengths are as follows.

\begin{tabular}{|l|l|l|}
\hline If $s<t \leq x:$ & $\ell_{s t}= \begin{cases}0, & \text { if } f(s) \geq t ; \\
t-f(s), & \text { if } t>f(s) ;\end{cases}$ & $\ell_{t s}=0 ;$ \\
\hline If $s \leq x<t:$ & $\ell_{s t}=f(t)-f(s) ;$ & $\ell_{t s}=0 ;$ \\
\hline If $x<s<t:$ & $\ell_{s t}=f(t)-f(s) ;$ & $\ell_{t s}= \begin{cases}f(s)-f(t), & \text { if } f(t)<s ; \\
f(s)-s, & \text { if } s \leq f(t) . \\
\hline\end{cases}$
\end{tabular}

Implementability can be verified in a manner similar to case $(i)$. It can be checked that splitting forward arcs can only decrease the cycle length. When removing sub-cycles consisting of a backward arc $(t, s)$, for $s<t$, together with the corresponding forward arcs, there are different cases. If $x<s<t$, the cycle length is non-negative by arguments similar to case $(i)$. If $s<x<t$ or $s<t<x$, the length of the backward arc is 0 and all forward arcs have non-negative length. Thus, such a sub-cycle has non-negative length and the argument can be continued as in $(i)$.

For revenue equivalence, we consider two cases. If $s<t \leq x$ or $x<s<t$, both $s$ and $t$ lie on a 0-length cycle just like in cases $(i)$ and $(i i)$. Hence, $\operatorname{dist}_{T_{f}}(s, t)+\operatorname{dist}_{T_{f}}(t, s)=0$. If $s \leq x<t$, an analogue argument as in $(i i)$ yields $d_{i s t_{T_{f}}}(s, x)=0$. With $\ell_{x t}=f(t)-f(x)$, we get dist $T_{f}(s, t) \leq f(t)-f(x)$. Similarly as in $(i)$, we can show that $\operatorname{dist}_{T_{f}}(t, x) \leq f(x)-f(t)$. Consequently, and since $\ell_{x s}=0$, we have $\operatorname{dist}_{T_{f}}(t, s) \leq \operatorname{dist}_{T_{f}}(t, x)+\ell_{x s} \leq f(x)-f(t)$. Thus, $\operatorname{dist}_{T_{f}}(s, t)+\operatorname{dist}_{T_{f}}(t, s) \leq 0$ and by implementability, $\operatorname{dist}_{T_{f}}(s, t)+\operatorname{dist}_{T_{f}}(t, s)=0$. Hence revenue equivalence holds.

Corollary 3 For the demand rationing problem, the proportional allocation rule $f$ with $f_{i}(t)=t_{i} / \sum_{j=1}^{n} t_{j}$ for $i=1, \ldots, n$ is implementable and satisfies revenue equivalence.

Proof. For every agent $i$ and every report of the other agents $t_{-i}$, the function $f_{i}\left(t_{i}, t_{-i}\right)=$ $t_{i} / \sum_{j=1}^{n} t_{j}$ satisfies the assumptions of either case $(i)$ or $(i i i)$ in Theorem 6. 
The proportional allocation rule completely distributes the supply among the retailers. In particular, a retailer can get more than his demanded quantity. In an economy without free disposal this may not be desired. In this case, the proportional allocation rule becomes $f_{i}(t)=\min \left\{t_{i}, t_{i} / \sum_{j=1}^{n} t_{j}\right\}$ for $i=1, \ldots, n$, and the supplier keeps part of the good if the supply exceeds the total demand. We next show that this allocation rule does not satisfy revenue equivalence. The result follows from the following Theorem.

Theorem 7 Suppose in the demand rationing problem, $f$ is implementable and there is one agent $i$ and reports $t_{-i}$ with $f_{i}\left(\cdot, t_{-i}\right)$ increasing and continuous such that there are $x_{1}, x_{2} \in$ $[0,1]$ with

$$
\begin{aligned}
f_{i}\left(t_{i}, t_{-i}\right) & >t_{i} \text { if } t_{i}<x_{1} \\
f_{i}\left(t_{i}, t_{-i}\right) & =t_{i} \text { if } x_{1} \leq t_{i} \leq x_{2} \\
f_{i}\left(t_{i}, t_{-i}\right) & <t_{i} \text { if } x_{2}<t_{i} .
\end{aligned}
$$

Then $f$ does not satisfy revenue equivalence.

Proof. Fix agent $i$ and report vector $t_{-i}$ of the other agents satisfying the assumptions of the Theorem. Regard the corresponding type graph $T_{f}$. There are six types of $\operatorname{arcs}(s, t)$ with $s<t$ depending on the position of $s$ and $t$ with respect to $x_{1}$ and $x_{2}$. Similarly, there are six types of $\operatorname{arcs}(t, s)$. It can be checked that the arc lengths are as follows.

\begin{tabular}{|l|l|l|}
\hline$s<t \leq x_{1}:$ & $\ell_{s t}= \begin{cases}0, & \text { if } f(s) \geq t ; \\
t-f(s), & \text { if } t>f(s) ;\end{cases}$ & $\ell_{t s}=0$ \\
\hline$x_{2} \leq s<t:$ & $\ell_{s t}=f(t)-f(s)$ & $\ell_{t s}= \begin{cases}f(s)-f(t), & \text { if } f(t)<s ; \\
f(s)-s, & \text { if } s \leq f(t) .\end{cases}$ \\
\hline all other cases: & $\ell_{s t}=f(t)-f(s)$ & $\ell_{t s}=0$ \\
\hline
\end{tabular}

Note that the only arcs with negative lengths are $(t, s)$-arcs for $x_{2} \leq s<t$.

That $f$ is implementable can be verified in a manner to the other cases. Splitting forward arcs can only reduce the length of a cycle. In order to verify that the total length of a subcycle consisting of a backward arc together with the corresponding forward arcs is indeed non-negative, one can check the case $x_{2} \leq s<t$ similar to case $(i)$ of Theorem 6 . In all other cases it is sufficient to note that the lengths of all involved forward and backward arcs are non-negative. 
We show that $f$ violates revenue equivalence by proving the existence of types $s$ and $t$ such that $\operatorname{dist}_{T_{f}}(s, t)+$ dist $_{T_{f}}(t, s)>0$. In fact, this inequality holds for any $s, t \in\left[x_{1}, x_{2}\right]$. Let $s, t \in\left[x_{1}, x_{2}\right], s<t$. Since $\ell_{t s}=0$, we get $\operatorname{dist}_{T_{f}}(t, s) \leq 0$. If $d_{i s t_{T_{f}}}(t, s)$ was in fact smaller than 0 , there would exist a path $p$ of negative length from $t$ to $s$. Such a path must contain at least one $\left(t^{\prime}, s^{\prime}\right)$-arc with $x_{2} \leq s^{\prime}<t^{\prime}$. In this case, the path must contain a sub-path from $t$ to $t^{\prime}$. Splitting forward arcs of this sub-path at $s^{\prime}$ (if necessary) yields a nonnegative sub-cycle through $s^{\prime}$ and $t^{\prime}$. The split does not increase the path length. Removing the sub-cycle can only reduce the length of $p$. This way, all negative arcs can be removed from $p$ without increasing its length. That contradicts the length of $p$ being negative. Hence, $\operatorname{dist}_{T_{f}}(t, s)=0$.

We claim that $\operatorname{dist}_{T_{f}}(s, t)=t-s>0$. To that end, note that any $(s, t)$-path that contains a backward arc, can be turned into a path of smaller or equal length without any backward arcs as follows. Suppose that backward arc $\left(t^{\prime}, s^{\prime}\right)$ is contained in $p, s^{\prime}<t^{\prime}$. Because $p$ is an $(s, t)$-path for $t>s$, it has to contain forward arcs that completely "pass" the $\left(t^{\prime}, s^{\prime}\right)$ arc in forward direction. Splitting all forward arcs passing $s^{\prime}$ at $s^{\prime}$ and splitting all forward arcs passing $t^{\prime}$ at $t^{\prime}$ does not increase $p$ 's length. Again, this splitting procedure results in a sub-cycle consisting of the $\left(t^{\prime}, s^{\prime}\right)$-arc and a forward path from $s^{\prime}$ to $t^{\prime}$. This sub-cycle has non-negative length and can be removed without increasing the length of $p$. Hence, we can without loss of generality restrict our attention to $(s, t)$-paths that contain only forward arcs. Such paths can only contain nodes from $[s, t] \subseteq\left[x_{1}, x_{2}\right]$. Therefore, the length of all such paths is equal to $f(t)-f(s)=t-s>0$, which proves the claim.

Corollary 4 For the demand rationing problem, the allocation rule $f$ with $f_{i}(t)=\min \left\{t_{i}\right.$, $\left.t_{i} / \sum_{j=1}^{n} t_{j}\right\}$ for $i=1, \ldots, n$ is implementable, but does not satisfy revenue equivalence.

Proof. For any agent $i$, and any $t_{-i}$, let $d_{-i}=\sum_{j \neq i} t_{j}$ be the total demand of the other agents. If $d_{-i}>1, f_{i}$ satisfies the assumptions of case $(i)$ of Theorem 6 . If $d_{-i}<1, f$ satisfies the assumptions of Theorem 7 for $x_{1}=0$ and $x_{2}=1-d_{-i}$. Implementability follows from the mentioned theorems. However revenue equivalence does not hold according to Theorem 7 . 


\section{Other Notions of Incentive Compatibility}

Our results can be extended to other notions of incentive compatibility. We briefly discuss two of them.

Ex-post incentive compatibility with externalities. The notation used throughout this paper can be used to model allocational externalities in auction settings, as allocations of items to other agents can be described by an extended set of outcomes $A$. In order to account for informational externalities in the case of ex-post incentive compatibility, the valuation function of agent $i$ is also a function of the true types of the other agents. An ex-post equilibrium is a Nash-equilibrium where every agent knows the types of all other agents. Formally, truth-telling is an ex-post equilibrium for allocation rule $f$ and payment scheme $\pi$ if for all agents $i$, all types $t_{i}$ and $s_{i}$ of $i$ and all types $t_{-i}$ of the other agents

$$
v_{i}\left(f\left(t_{i}, t_{-i}\right), t_{i}, t_{-i}\right)-\pi\left(t_{i}, t_{-i}\right) \geq v_{i}\left(f\left(s_{i}, t_{-i}\right), t_{i}, t_{-i}\right)-\pi\left(s_{i}, t_{-i}\right) .
$$

Again, incentive compatible payment schemes can be associated with node potentials in the allocation graphs. The arc lengths in $G_{f}$ have to be defined as follows:

$$
\ell_{a b}=\inf _{t_{i} \in f^{-1}(b)}\left(v_{i}\left(b, t_{i}, t_{-i}\right)-v_{i}\left(a, t_{i}, t_{-i}\right)\right) .
$$

We get the following result.

Theorem 8 An ex-post implementable allocation rule $f$ satisfies revenue equivalence if and only if in every allocation graph $G_{f}$, for every two allocations $a, b, \operatorname{dist}_{G_{f}}(a, b)+\operatorname{dist}_{G_{f}}(b, a)=$ 0 .

Bayes-Nash incentive compatibility. Müller, Perea, and Wolf (2007) already used type graphs for the case of Bayes-Nash incentive compatible allocation rules. In this setting, the (multi-dimensional) types of all agents are independently distributed. The notation used by Müller et al. accounts for allocational as well as informational externalities, that is, the valuation of an agent depends on the chosen allocation, the type of the agent and the types of all other agents.

An allocation rule $f$ is said to be Bayes-Nash incentive compatible if there is a payment 
scheme $\pi$ such that for all agents $i$ and all types $t_{i}$ and $s_{i}$ of $i$ the following is true

$$
E_{-i}\left[v_{i}\left(f\left(t_{i}, t_{-i}\right), t_{i}, t_{-i}\right)-\pi_{i}\left(t_{i}, t_{-i}\right)\right] \geq E_{-i}\left[v_{i}\left(f\left(s_{i}, t_{-i}\right), t_{i}, t_{-i}\right)-\pi_{i}\left(s_{i}, t_{-i}\right)\right]
$$

where the expected value is taken with respect to the types of all agents other than $i$. That is, truth-telling of every agent is a Nash equilibrium when agents try to maximize expected utilities. We use the type graph with arc lengths defined as follows:

$$
\ell_{s_{i} t_{i}}=E_{-i}\left[v_{i}\left(f\left(t_{i}, t_{-i}\right), t_{i}, t_{-i}\right)-v_{i}\left(f\left(s_{i}, t_{-i}\right), t_{i}, t_{-i}\right)\right] .
$$

Note that by taking expectations, there is a single type graph for every agent. Here, we analyze expected payment schemes $E_{-i}\left(\pi_{i}\left(\cdot, t_{-i}\right)\right)$, and an allocation rule satisfies revenue equivalence if expected payments are unique up to a constant.

Theorem 9 A Bayes-Nash implementable allocation rule $f$ satisfies revenue equivalence with respect to expected payments if and only if in every type graph $T_{f}$ for all types s and $t$ of this agent, dist $_{T_{f}}(s, t)+\operatorname{dist}_{T_{f}}(t, s)=0$.

Incentive compatibility, monotonicity and potential functions. In settings with multi-dimensional type spaces, finite $A$, and valuation functions that are linear in types, it was established by several authors that implementability, and thus the non-negative cycle property, is sometimes implied by a monotonicity property, termed weak monotonicity in Bikhchandani, Chatterjee, Lavi, Mu'alem, Nisan, and Sen (2006). For dominant strategy incentive compatibility see Bikhchandani et al., Gui, Müller, and Vohra (2004), Saks and Yu (2005), and Monderer (2007), for Bayes-Nash incentive compatibility see Müller, Perea, and Wolf (2007). In these settings implementability implies that the allocation rule $f$, viewed from a single agent perspective as a vector field that maps multi-dimensional types on lotteries over outcomes, has a potential function $F$. One can easily verify that this property has the following interpretation on type graphs: the length of a shortest path in $T_{f}$ from some type $s$ to some type $t$ is upper bounded by a path integral of the vector field $f$, or equivalently $F(t)-$ $F(s)$. From this it follows easily that $\operatorname{dist}_{T_{f}}(s, t)=-\operatorname{dist}_{T_{f}}(t, s)$, i.e. revenue equivalence holds. In particular, dist $_{T_{f}}(s, t)=F(t)-F(s)$ for any potential function $F$. This connection between implementability, potential functions and revenue equivalence is also established in Jehiel and Moldovanu (2001) and Krishna and Maenner (2001). 


\section{Acknowledgements}

We thank the participants of Dagstuhl Seminars 07271 and 07471 for their valuable feedback.

\section{References}

Bikhchandani, S., S. Chatterjee, R. Lavi, A. Mu'alem, N. Nisan, and A. Sen (2006). Weak monotonicity characterizes deterministic dominant strategy implementation. Econometrica $74(4), 1109-1132$.

Cachon, G. and M. Lariviere (1999). Capacity choice and allocation: Strategic behavior and supply chain performance. Management Science 45(8), 1091-1108.

Chung, K.-S. and W. Olszewski (2007). A non-differentiable approach to revenue equivalence. Forthcoming in Theoretical Economics, www.econtheory.org.

Green, J. and J.-J. Laffont (1977). Characterization of satisfactory mechanisms for the revelation of preferences for public goods. Econometrica 45(2), 427-438.

Gui, H., R. Müller, and R. Vohra (2004, October). Dominant strategy mechanisms with multidimensional types. Discussion Paper 1392, The Center for Mathematical Studies in Economics \& Management Sciences, Northwestern University, Evanston, IL.

Holmström, B. (1979). Groves' scheme on restricted domains. Econometrica 47(5), 11371144.

Jehiel, P. and B. Moldovanu (2001). Efficient design with interdependent valuations. Econometrica $69(5), 12371259$.

Klemperer, P. (1999). Auction theory: A guide to the literature. Journal of Economic Surveys 13(3), 227-286.

Krishna, V. (2002). Auction Theory. Academic Press.

Krishna, V. and E. Maenner (2001). Convex potentials with an application to mechanism design. Econometrica 69(4), 1113-1119.

Milgrom, P. and I. Segal (2002). Envelope theorems for arbitrary choice sets. Econometrica $70(2), 583-601$.

Monderer, D. (2007). Monotonicity and implementability. Discussion paper, Technion, Haifa. 
Müller, R., A. Perea, and S. Wolf (2007). Weak monotonicity and Bayes-Nash incentive compatibility. Games and Economic Behavior. To appear. Available as METEOR research memorandum RM/05/039.

Myerson, R. (1981). Optimal auction design. Mathematics of Operations Research 6(1), $58-73$.

Rochet, J.-C. (1987). A necessary and sufficient condition for rationalizability in a quasilinear context. Journal of Mathematical Economics 16(2), 191-200.

Saks, M. and L. Yu (2005). Weak monotonicity suffices for truthfulness on convex domains. In Proc. 6th ACM conference on Electronic Commerce, pp. 286 - 293.

Schrijver, A. (2003). Combinatorial Optimization, Volume A. Springer.

Suijs, J. (1996). On incentive compatibility and budget balancedness in public decision making. Economic Design 2, 193-209. 